\title{
Strategies for implementation of guideline recommended cardiovascular risk management for patients with rheumatoid arthritis: results from a questionnaire survey of expert rheumatology centers
}

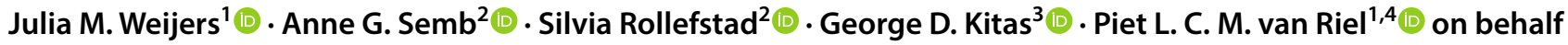 \\ of ATrans-Atlantic Cardiovascular Consortium for Rheumatoid Arthritis (ATACC-RA)
}

Received: 22 December 2019 / Accepted: 8 February 2020 / Published online: 22 February 2020

(c) The Author(s) 2020

\begin{abstract}
The aim was to study the different strategies used to implement cardiovascular risk evaluation and management for patients with rheumatoid arthritis (RA) in daily clinical practice. A questionnaire survey was performed among both the members of the international Trans-Atlantic Cardiovascular Risk Consortium for Rheumatoid Arthritis (ATACC-RA) as well as the Survey of cardiovascular disease risk factors (CVD-RF) in patients with RA (SURF-RA) group. The questionnaire included 18 questions with the overarching topics: (1) organization and responsibility of cardiovascular risk management (CVRM); (2) screening of CVD-RFs; (3) overview current CVRM status; and (4) availability of data regarding CVRM. Based on the answers, two researchers (JW, PR) independently categorized the different strategies. Thirteen out of 27 rheumatology centers responded to the questionnaire. One rheumatology center did not have organized CVRM for their RA patients. Among the other centers, three strategies to organize CVRM in daily practice were distinguished: (1) the rheumatologist performs CVRM during outpatient visits $(n=6)$; (2) cardiologists and rheumatologists co-operate in a cardio-rheuma-clinic/team with different tasks and responsibilities $(n=3)$; and (3) the general practitioner screens and intervenes on CVD-RFs $(n=3)$. Each CVRM strategy was based on agreements between medical professionals and was also dependent on the national healthcare system and available financial resources. Three strategies were identified for CVRM implementation in daily clinical practice based on who is primarily responsible for performing CVRM. More research is warranted to compare their relative merits and effectiveness in relation to CVRM.
\end{abstract}

Keywords Cardiovascular diseases · Guideline adherence · Organization · Rheumatoid arthritis

Electronic supplementary material The online version of this article (https://doi.org/10.1007/s00296-020-04533-4) contains supplementary material, which is available to authorized users.

Julia M. Weijers

julia.weijers@ radboudumc.nl

Anne G. Semb

a-semb@diakonsyk.no

Silvia Rollefstad

sc.rollefstad@gmail.com

George D. Kitas

george.kitas@nhs.net

Piet L. C. M. van Riel piet.vanriel@radboudumc.nl
1 Scientific Center for Quality of Healthcare, IQ Healthcare, Radboud university medical center, Radboud Institute for Health Sciences, PO box 9101 (114), 6500 HB Nijmegen, The Netherlands

2 Department of Rheumatology, Preventive Cardio-Rheuma Clinic, Diakonhjemmet Hospital, Oslo, Norway

3 Department of Rheumatology, Dudley Group NHS Foundation Trust, West Midlands, Dudley, United Kingdom

4 Department of Rheumatology, Bernhoven, Uden, The Netherlands 


\section{Introduction}

It has already been known for decades that patients with rheumatoid arthritis (RA) have an increased risk of cardiovascular diseases (CVD), such as myocardial infarctions, cerebrovascular accidents, and congestive heart failures compared with the general population [1-3]. Despite this, not much attention has been given to this patient group regarding CVD prevention $[4,5]$.

Therefore international guidelines have been developed, such as the guidelines of the American College of Cardiology (ACC) and American Heart Association (AHA) [6-8], the European Society of Cardiology (ESC) guidelines for cardiovascular risk management (CVRM) [9, 10], and the European League Against Rheumatism (EULAR) recommendations for CVRM in patients with RA and other forms of inflammatory joint diseases $[11,12]$. These guidelines state that besides optimal control of disease activity to lower CVD risk, regularly CVD riskassessment is recommended for all patients with RA. The present assessment of CVRM consists of screening of five traditional CVD risk factors (CVD-RFs): blood pressure, blood glucose, lipid profile, smoking status and body weight. Following the assessment of CVD-RFs, CVD preventive treatment should be initiated, if indicated. In addition to pharmacological therapy, lifestyle modification including a healthy diet, physical activity and smoking cessation should be supported [12].

These CVRM guidelines, and guidelines in general, are intended to improve the quality of care. They are mainly written to make the rapidly growing (scientific) information flow manageable; to reduce variation in actions between healthcare providers; to base clinical actions more on scientific evidence and to work more transparently [13]. However, a literature search indicates that CVRM guidelines as well as guidelines in general are not applied effectively in routine clinical practices [14-16]. Percentages of patients with RA of whom CVD-RFs are identified vary between 24.9 and $88 \%$ [15, 17]. This variation in guideline adherence may be related to the different strategies used to implement CVRM in daily care. CVRM can be improved when rheumatology centers could learn from each other by comparing their daily CVRM care with others who have proved to work according to the recommendations. However, until now, it is unknown which approaches are used to organize CVRM according to the recommendations. This questionnaire survey is the first step to clarify this variation by identifying various systems for CVD risk evaluation and management in patients with RA in rheumatology expert centers across the world.

\section{Methods}

To evaluate differences in the organization of CVRM in patients with RA, we took advantage of two already existing networks in rheumatology. A questionnaire covering aspects of CVRM was sent by an email in 2017 to all members of both A Trans Atlantic CVD risk Consortium for patients with RA (ATACC-RA, www.atacc-ra.com), and to the Survey of CVD Risk Factors in patients with RA (SURF-RA) audit group. ATACC-RA was formed in 2013 and includes 18 rheumatology centers from 13 countries [18]. The main aim of the ATACC-RA is to evaluate the burden of CVD in rheumatic and musculoskeletel diseases (RMD) to increase awareness of CVD and to develop implementation strategies to improve the care for patients with RMD. The SURF-RA is a large international survey including centers from 19 countries across the world that systematically examines the recording and control of CVD-RFs in patients with RA [18]. Topics covered in the questionnaire (see supplementary material 1) sent out to the centers were: (1) organization and responsibility of CVRM; (2) screening of traditional CVD-RF; (3) current CVRM status; and (4) availability of data regarding CVRM. Independent of each other, one researcher (JW) and one rheumatologist (PR), read the returned questionnaires and categorized clinics based primarily on which healthcare professionals were involved in CVRM. Afterwards, they discussed their findings and reached consensus about the different categories. This study was submitted to the local Ethics Committee Arnhem-Nijmegen and it was judged that the study did not fall within the scope of the Medical Research Involving Human Subjects Act (number 2020-6133). To ensure the privacy of the respondents, researchers worked with anonymized data. Furthermore, it was guaranteed that the data were not published with recognizable rheumatology centers.

\section{Results}

\section{Participating centers}

In total, 13 members of the ATACC-RA or SURF-RA from Europe, North America, South America and Asia returned the questionnaire, which corresponds to a response rate of $48 \%$ (13/27). Although $9.5 \%$ of the data were missing, it was possible to identify the strategy used to organize CVRM in all respondents. On average, each rheumatology center had a population of $1668(\min 150, \max 5000)$ patients with RA. Six out of 13 respondents reported that their outpatient clinic has procedures according to 
an (inter)national guideline for CVRM, with the 2016 CVRM EULAR recommendation most frequently used [12]. Two other respondents reported that they had agreements between healthcare professionals [rheumatologists and general practitioners (GPs)]: these agreements were derived from the EULAR recommendations [12] or European Society of Cardiology guidelines for CVD prevention [19]. The remaining five respondents reported that they had standard operating procedures related to CVRM, without alluding to any published recommendations. Nine rheumatology centers reported to have (digital) data related to CVRM as a part of their clinical care routine. However, the majority did not have data of their total RA population. In most centers, data were limited to a subgroup of the total RA population.

\section{Strategies to implement guideline recommended CVRM in daily practice}

Three different strategies were distinguished to deliver CVRM care, and explained below in Fig. 1. One out of the 13 centers did not have organized CVRM for patients with RA in their daily practice.

\section{The rheumatologist is responsible for adequate CVRM} (Fig. 1a)

Six rheumatology centers reported that the treating rheumatologist is primarily responsible for identification, diagnosis, treatment, and follow-up of CVD-RFs in patients with RA. Screening and decision making about (non)pharmacological treatment of potential CVD-RFs take place during rheumatology outpatient clinic consultations. Whether and how frequently assessment of CVD-RFs is performed is dependent on each individual rheumatologist. This strategy required no specific agreements between the rheumatologist and other health care professionals related to CVRM (e.g.
GPs), because the rheumatologist is solely responsible for all stages of CVRM.

\section{Cooperation in a cardio-rheuma-clinic/team with shared responsibilities (Fig. 1b)}

In three rheumatology centers the cardiologists, rheumatologists and sometimes also a general practitioner (GP) cooperate in a cardio-rheuma-clinic with different tasks and responsibilities. CVD-RFs are identified and recorded by the rheumatologist or rheumatology specialist nurses. Patients with a high risk of CVD are referred to a cardiologist within the same hospital or a surrounding rheumatology center. CVRM is performed according to agreements between the different healthcare professionals who are involved in the different stages of CVRM. Information about CVD-RFs and related treatments is recorded in the medical records and the information is exchanged via letters, in many cases electronic.

\section{The rheumatologist and GP collaborate closely to manage CVRM (Fig. 1c)}

In the remaining three rheumatology centers, primary and secondary care professionals work together, to perform CVRM in the relevant health district. The first step, which is performed by the rheumatology team, is to identify patients with an increased risk of CVD eligible for the CVRM care program. The patients are referred to the GP for further CVD-RF recording and implementation of CVD risk preventive measures as pharmacological and lifestyle interventions, or onwards referral to a secondary care cardiology service. The exact tasks and responsibilities of the health care professionals working in primary and secondary care are arranged in regional transmural appointments. Communication about the current CVRM status will be performed by (electronic) letter or a digital patient portal.
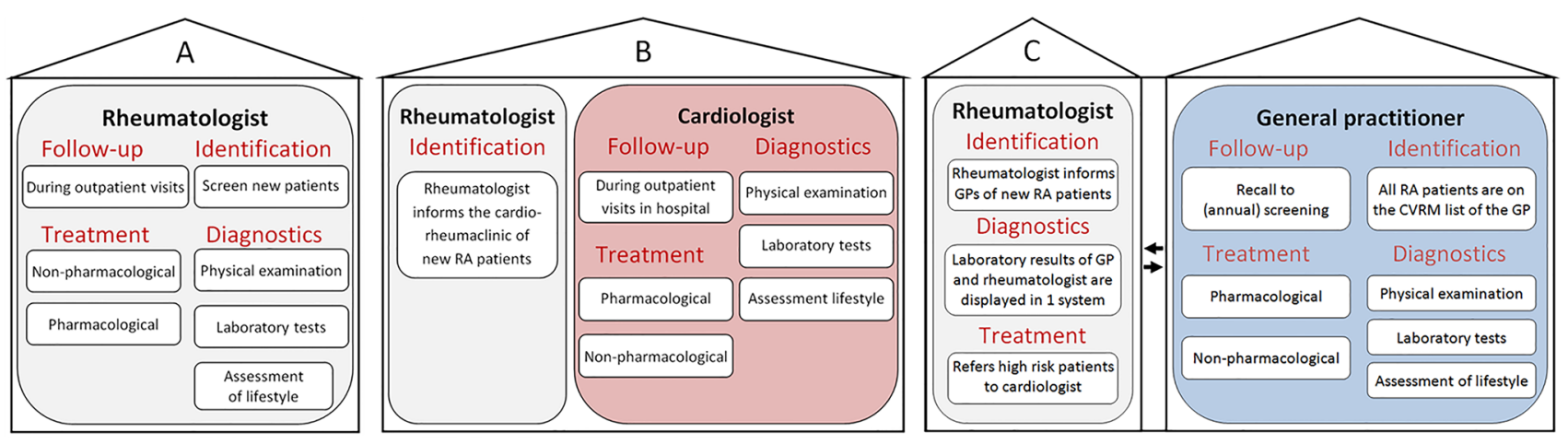

Fig. 1 Three strategies for implementation of cardiovascular risk management in daily practice 


\section{Discussion}

This report describes three different strategies to implement guideline recommended CVRM for patients with RA in daily care. Adequate CVRM implies screening, appropriate lifestyle and pharmacological treatment of identified CVD-RFs to specific targets and follow-up to confirm that targets have been reached. Screening for the presence of CVD-RFs is not sufficient in itself, without sufficient subsequent action, to reduce the increased risk of CVD in patients with RA. As with the majority of studies, the design of the current study also contains limitations. The first limitation is that this study does not contain information on the outcomes of CVRM as this was outside the scope of this survey. Our survey was confined to identify the systems of CVRM and did not contain information on the results of CVRM. Due to practical (e.g. data stored in different software programs or only applicable on paper) and ethical reasons, it was not possible for the respondents of the survey to share their data related to results of CVRM. However, to our knowledge this is the first study that focused on the organization of care related to CVRM in patients with RA.

In both United States and Europe, patients with RA receive suboptimal care for their increased risk of CVD $[15,17]$. Members of the ATACC-RA group are presumably particularly motivated and interested in CVRM, and the majority of respondents had a system for CVRM in place and followed international guidelines for CVRM. Almost all respondents reported that they performed screening of CVD-RFs at the first rheumatology outpatient consultation or soon after the diagnosis of RA was established. However, it is also important that both CVDRFs assessment and intervention outcomes are regularly monitored and evaluated. In some rheumatology centres, there may be well room for improvement in follow-up of CVD-RFs assessment after screening.

The second limitation is the relatively low number of rheumatology centers that responded to the questionnaire. This might have an impact on the representativeness of the results. However, saturation of the answers to the questions was reached and each strategy to organize CVRM was reported at least thrice. Therefore, it is unlikely that a major strategy for CVRM was missed.

To date, it remains unclear which strategy results in the best adherence to international guidelines on CVRM. To compare the effectiveness of the various strategies of CVRM, data about CVD-RFs, treatment and outcomes are necessary. The overarching principle in the 2016 EULAR recommendations for CVD risk management is that the rheumatologist is responsible for CVD risk management in patients with RA and other inflammatory rheumatic diseases [12]. We advocate to optimize CVRM and to compare various strategies, a systematic recording of CVD-RF screening, treatment and targets in the medical files should be introduced in each rheumatology outpatient clinic and comparative audits could be performed. To a certain extent, this task is currently being undertaken by the international SURF-RA audit (www.atacc-ra.com). The impact of national health care system configuration and resources available to achieve CVRM must also be accurately assessed as this might have an impact on the results as well.

Independent of how CVRM is organized, adequate communication and feedback between the medical professionals involved and preferably also with the patients are of paramount importance. Each strategy may have advantages and disadvantages in this respect. In strategies involving the rheumatology team being solely responsible for CVRM, communication with other health professionals may appear of less importance, but the team must be aware of their knowledge and expertise limitations regarding CVRM and seek appropriate advice or onward referral when necessary. Strategies involving combined cardio-rheuma clinics have obvious potential advantages in terms of interdisciplinary communication and available expertise. However, in some countries or rheumatology centers, this strategy might be not possible due to either financial or staff shortage. Cardiorheuma clinics may not be feasible in all healthcare systems and their clinical efficacy needs to be balanced against healthcare costs. Effective communication is of extreme importance in strategies where each healthcare professional (i.e. rheumatology, primary care and cardiology) has distinct responsibilities.

Independent of the various strategies developed by the different health care teams, relevant patient education may enable patients to play an active role by directly discussing the importance of adequate CVRM with their caregivers and initiate appropriate CVRM [19]. This may also have additional benefits where lifestyle modification is concerned [20].

\section{Conclusion}

This study identified three strategies to implement these CVRM in daily clinical practice based on who is primarily performing CVRM: the rheumatologist, a cardio-rheumaclinic/team or the GP. Their relative merits and drawbacks should be prospectively assessed in future studies.

Acknowledgements The authors would thank the members of ATTACRA and the participating centers of the SURF-RA study who discussed with us about the design of the survey and who respond on the questionnaire about the organization of CVRM in their clinic: P. Dessein (Brussels, Belgium), D. Galarza-Delgado (San Nicolás de los Garza, 
Mexico), M. González-Gay (Santander, Spain), C. Hitchon (Winnipeg, Canada), B. Kuriya (Toronto, Canada), D. Misra (Lucknow, India), E. Mirrakhimov (Bishkek, Kyrgyzstan), E. Myasoedova (Rochester, United States), V. Ramos (Mexico City, Mexico) S. Wållberg-Jonsson (Umeå, Sweden).

Author contribution All authors contributed to the study conception and design. Material preparation, data collection and analysis were performed by JW and PR. The first draft of the manuscript was written by JW, AGS and PR and all authors commented on previous versions of the manuscript. All authors read and approved the final manuscript.

Funding There was no financial support for this study by any company or organization.

\section{Compliance with ethical standards}

Conflict of interest JW, SR, GK and PR declare that they have no conflict of interest. AS received personal fees as speaker honoraria/ consulting fees from Merck/Schering-Plough, AbbVie, Bayer, UCB, Novartis, BMS, Lilly, Sanofi Aventis, and Lilly, and unrestricted collaboration research grant to Diakonhjemmet Hospital from Lilly.

Open Access This article is licensed under a Creative Commons Attribution 4.0 International License, which permits use, sharing, adaptation, distribution and reproduction in any medium or format, as long as you give appropriate credit to the original author(s) and the source, provide a link to the Creative Commons licence, and indicate if changes were made. The images or other third party material in this article are included in the article's Creative Commons licence, unless indicated otherwise in a credit line to the material. If material is not included in the article's Creative Commons licence and your intended use is not permitted by statutory regulation or exceeds the permitted use, you will need to obtain permission directly from the copyright holder. To view a copy of this licence, visit http://creativecommons.org/licenses/by/4.0/.

\section{References}

1. Avina-Zubieta JA, Thomas J, Sadatsafavi M, Lehman AJ, Lacaille D (2012) Risk of incident cardiovascular events in patients with rheumatoid arthritis: a meta-analysis of observational studies. Ann Rheum Dis 71(9):1524-1529

2. Watson DJ, Rhodes T, Guess HA (2003) All-cause mortality and vascular events among patients with rheumatoid arthritis, osteoarthritis, or no arthritis in the UK General Practice Research Database. J Rheumatol 30(6):1196-1202

3. Situnayake RD, Kitas G (1997) Dyslipidemia and rheumatoid arthritis. Ann Rheum Dis 56(6):341-342

4. Desai SS, Myles JD, Kaplan MJ (2012) Suboptimal cardiovascular risk factor identification and management in patients with rheumatoid arthritis: a cohort analysis. Arthritis Res Ther 14(6):R270

5. Barber CE, Esdaile JM, Martin LO, Faris P, Barnabe C, Guo S et al (2016) Gaps in addressing cardiovascular risk in rheumatoid arthritis: assessing performance using cardiovascular quality indicators. J Rheumatol 43(11):1965-1973

6. Stone NJ, Robinson JG, Lichtenstein AH, Bairey Merz CN, Blum CB, Eckel RH et al (2014) 2013 ACC/AHA guideline on the treatment of blood cholesterol to reduce atherosclerotic cardiovascular risk in adults: a report of the American College of Cardiology/ American Heart Association Task Force on Practice Guidelines. J Am Coll Cardiol 63(25 Pt B):2889-2934

7. Arnett DK, Blumenthal RS, Albert MA, Buroker AB, Goldberger ZD, Hahn EJ et al (2019) 2019 ACC/AHA guideline on the primary prevention of cardiovascular disease: a report of the American College of Cardiology/American Heart Association Task Force on Clinical Practice Guidelines. Circulation 140(11):e596-e646

8. Grundy SM, Stone NJ, Bailey AL, Beam C, Birtcher KK, Blumenthal RS et al (2019) 2018 AHA/ACC/AACVPR/AAPA/ABC/ ACPM/ADA/AGS/APhA/ASPC/NLA/PCNA guideline on the management of blood cholesterol: a report of the American College of Cardiology/American Heart Association Task Force on Clinical Practice Guidelines. Circulation 139(25):e1082-e1143

9. Catapano AL, Graham I, De Backer G, Wiklund O, Chapman MJ, Drexel H et al (2016) 2016 ESC/EAS guidelines for the management of dyslipidaemias. Eur Heart J 37(39):2999-3058

10. Mach F, Baigent C, Catapano AL, Koskinas KC, Casula M, Badimon L et al (2019) 2019 ESC/EAS Guidelines for the management of dyslipidaemias: lipid modification to reduce cardiovascular risk. Eur Heart J 41(1):111-188

11. Peters MJ, Symmons DP, McCarey D, Dijkmans BA, Nicola P, Kvien TK et al (2010) EULAR evidence-based recommendations for cardiovascular risk management in patients with rheumatoid arthritis and other forms of inflammatory arthritis. Ann Rheum Dis 69(2):325-331

12. Agca R, Heslinga SC, Rollefstad S, Heslinga M, McInnes IB, Peters MJ et al (2016) EULAR recommendations for cardiovascular disease risk management in patients with rheumatoid arthritis and other forms of inflammatory joint disorders: 2015/2016 update. Ann Rheum Dis 76(1):17-2813

13. Grimshaw JM, Russell IT (1993) Effect of clinical guidelines on medical practice: a systematic review of rigorous evaluations. Lancet 342(8883):1317-1322

14. Lesuis N, den Broeder AA, Hulscher ME, van Vollenhoven RF (2016) Practice what you preach? An exploratory multilevel study on rheumatoid arthritis guideline adherence by rheumatologists. RMD Open 2(1):e000195

15. Weijers JM, Rongen-van Dartel SAA, Hoevenaars D, Rubens M, Hulscher M, van Riel P (2018) Implementation of the EULAR cardiovascular risk management guideline in patients with rheumatoid arthritis: results of a successful collaboration between primary and secondary care. Ann Rheum Dis 77(4):480-483

16. Ladak K, Hashim J, Clifford-Rashotte M, Tandon V, Matsos M, Patel A (2018) Cardiovascular risk management in rheumatoid arthritis: a large gap to close. Musculoskelet Care 16(1):152-157

17. Schmidt TJ, Avina-Zubieta JA, Sayre EC, Abrahamowicz M, Esdaile JM, Lacaille D (2018) Quality of care for cardiovascular disease prevention in rheumatoid arthritis: compliance with hyperlipidemia screening guidelines. Rheumatology (Oxford) 57(10):1789-1794

18. ATTAC-RA (2018) A trans-atlantic cardiovascular consortium for rheumatoid arthritis Norway. Available from: https://atacc-ra. com. Accessed 3 Feb 2019

19. John H, Carroll D, Kitas GD (2011) Cardiovascular education for people with rheumatoid arthritis: what can existing patient education programmes teach us? Rheumatology (Oxford) 50(10):1751-1759

20. Veldhuijzen van Zanten JJ, Rouse PC, Hale ED, Ntoumanis N, Metsios GS, Duda JL et al (2015) Perceived barriers, facilitators and benefits for regular physical activity and exercise in patients with rheumatoid arthritis: a review of the literature. Sports Med 45(10):1401-1412

Publisher's Note Springer Nature remains neutral with regard to jurisdictional claims in published maps and institutional affiliations. 